\title{
Identification and Biosynthesis of Cholest-4-en-3-one and 6-Oxocholetanol in Young Tomato Plants
}

\author{
Sang Cheul Lee, Tae-Wuk Kim, Jung-Yun Hwang, Chan Ho Park, Seung-Hyun Son, Ji Hyun Youn, and Seong-Ki Kim*
}

\author{
Department of Life Science, Chung-Ang University, Seoul 156-756, Korea
}

Received February 11, 2010, Accepted April 8, 2010

Key Words: Brassinosteroids, $\mathrm{C}_{27}$-brassinosteroid biosynthesis, Young tomato plant

Polyhydroxylated plant steroids, collectively referred to as the brassinosteroids (BRs), are a new class of plant hormones that regulate a variety of phenomena in plant growth and development. ${ }^{1,2}$ Thus far, more than 40 BRs have been identified throughout the entirety of the plant kingdom. ${ }^{3,4}$ Naturallyoccurring BRs can be classified as $\mathrm{C}_{27^{-}}, \mathrm{C}_{28^{-}}$and $\mathrm{C}_{29}$-BRs on the basis of their carbon skeletons. The structural similarity between BRs and phytosterols suggests that BRs may be biosynthesized from phytosterols, which have the same carbon skeletons as BRs. ${ }^{5,6}$ In fact, bio-organic feeding experiments and molecular genetic studies have revealed that biologically active $\mathrm{C}_{28}$-BRs, castasterone (Fig. 1, CS) and brassinolide, are biosynthesized from campesterol (CR) by two parallel biosynthetic pathways designated the early and late C6-oxidation pathway--in many plants. ${ }^{7}$ Recently, we showed that young tomato plants exploit a novel biosynthetic pathway to generate a $\mathrm{C}_{27}$-BR, 28-norCS from cholesterol (CHR). ${ }^{7}$ The pathway for $\mathrm{C}_{27}$-BRs biosynthesis in the tomato plants was basically identical to that of the late C6-oxidation pathway for $\mathrm{C}_{28}$-BRs biosynthesis, thus indicating that the late $\mathrm{C} 6$-oxidation pathway for $\mathrm{C}_{27}$-BRs biosynthesis is functional in the plants. This finding compelled us to assess the possible operation of the early C6oxidation pathway for $\mathrm{C}_{27}$-BRs biosynthesis in the tomato plants. Toward this end, we attempted to identify biosynthetic precursors in the early C6-oxidation pathway for $\mathrm{C}_{27}$-BRs biosynthesis in young tomato plants, and characterize their metabolism.

The unsaponified lipid obtained from young tomato plants was purified with an $\mathrm{SiO}_{2}$ and a Sep-Pak C18 column chromatography. After reversed-phase HPLC (Senshu-Pak Pegasil-B, $4.6 \times 250 \mathrm{~mm})$, the fractions corresponding to authentic 6oxoCHN (18 - $19 \mathrm{~min}$ ) and cholest-4-en-3-one (37 - $39 \mathrm{~min})$ were collected, and then analyzed via capillary GC-MS. ${ }^{3}$

After 30 minutes of treatment of $N$-methyl- $N$-TMS-trifluroacetamide at $70{ }^{\circ} \mathrm{C}$, trimethylsillylic (TMSi) ether of a compound in HPLC fractions 18 and 19 yielded a molecular ion at $\mathrm{m} / \mathrm{z} 474$ and prominent ions at $\mathrm{m} / \mathrm{z} 459,445,384,221$ and 159 at the same GC retention time (19.51 $\mathrm{min})$ as that of authentic 6-oxoCHN TMSi ether (Table 1). Therefore, the compound was identified as 6-oxoCHN. Without derivatization, a compound in HPLC fraction 37 - 39 evidenced a molecular ion at $\mathrm{m} / \mathrm{z} 384$ and characteristic ions at $\mathrm{m} / \mathrm{z} 342,261,229$ and 124 with identical retention times on GC to that of synthetic cholest-4-en-3one; this finding confirmed the identification of the compound as cholest-4-en-3-one. Consequently, cholest-4-en-3-one and
6-oxoCHN were detected in the tomato plants and identified as potent intermediates involved in the early $\mathrm{C6}$-oxidation pathway for $\mathrm{C}_{27}$-BRs.

In order to confirm the involvement of cholest-4-en-3-one and 6-oxoCHN in the early C6-oxidation pathway for $\mathrm{C}_{27}$ - $\mathrm{BRs}$, in vitro enzymatic conversions of related sterols such as $\mathrm{CHR}$, cholest-4-en-3-one, and CHN were conducted. The reactions were initiated via the addition of substrates (CHR, cholest-4en-3-one and $\mathrm{CHN}$ ), followed by $30 \mathrm{~min}$ of incubation at $37^{\circ} \mathrm{C}$. The metabolites of the enzyme reactions were extracted with ethyl acetate, and purified by a Sep-Pak C18 cartridge and a reversed-phase HPLC. Finally, the enzyme products were

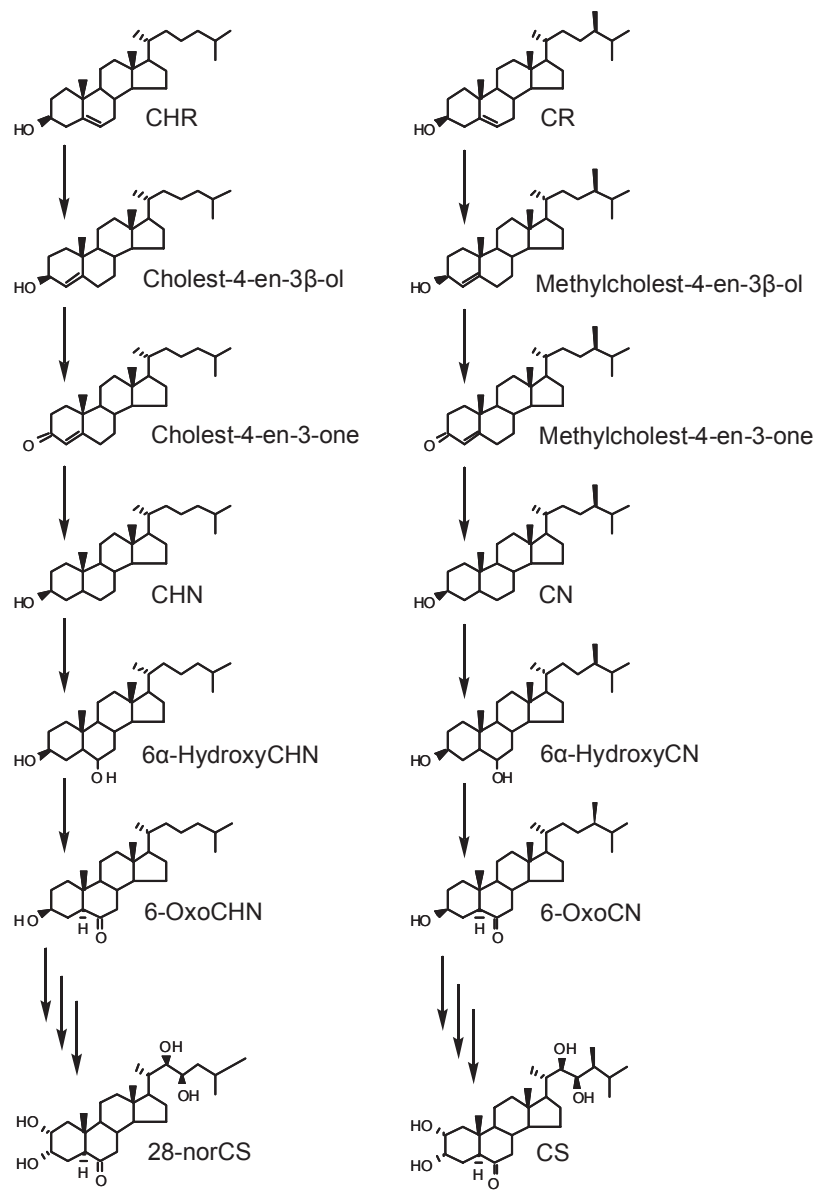

Figure 1. The upstream biosynthetic pathway for the early C6-oxidation pathway for $\mathrm{C}_{27}$ and $\mathrm{C}_{28}$-BRs biosynthesis. 
Table 1. GC-MS data for 6-oxoCHN and cholest-4-en-3-one in young tomato plants.

\begin{tabular}{lcl}
\hline Compound & $\mathrm{Rt}^{a}(\mathrm{~min})$ on GC & Prominent ions (relative intensity, \%) \\
\hline Tomato 6-oxoCHN & 19.51 & $474(\mathrm{M}+, 19), 459(51), 445(100), 384(4), 159(8)$ \\
Tomato cholest-4-en-3-one & 13.60 & $384(\mathrm{M}+, 58), 369(8), 342(22), 261(26), 229(42), 124(100)$ \\
Authentic 6-oxoCHN & 19.51 & $474(\mathrm{M}+, 18), 459(48), 445(100), 384(6), 159(10)$ \\
Authentic cholest-4-en-3-one & 13.60 & $384(\mathrm{M}+, 60), 369(8), 342(24), 261(24), 229(46), 124(100)$ \\
\hline
\end{tabular}

${ }^{a}$ Retention time. ${ }^{b}$ The samples were analyzed as a derivative of TMSi ether.

Table 2. Enzymatic conversion of CHR, cholest-4-en-3-one and CHN in young tomato plants.

\begin{tabular}{ccl}
\hline Substrate & \multicolumn{1}{c}{ Product } & Prominent ions (relative intensity, \%) \\
\hline CHR & Cholest-4-en-3-one & $384(\mathrm{M}+, 61), 369(9), 342(22), 261(25), 229(40), 124(100)$ \\
& $\mathrm{CHN}^{a}$ & $460(\mathrm{M}+, 54), 445(73), 370(28), 355(43), 305(33), 215(100)$ \\
Cholest-4-en-3-one & $\mathrm{CHN}^{a}$ & $460(\mathrm{M}+, 73), 445(90), 370(46), 355(56), 305(35), 215(100)$ \\
CHN & 6-OxoCHN & $474(\mathrm{M}+, 19), 459(51), 445(100), 384(4), 159(8)$
\end{tabular}

${ }^{a}$ The samples were analyzed as a derivative of TMSi ether.

analyzed via capillary GC-MS/SIM after suitable derivatization.

As summarized in Table 2, when CHR was added as a substrate, two products were identified: cholest-4-en-3-one and $\mathrm{CHN}$. When cholest-4-en-3-one was used, CHN was generated as a product. Thus, the presence of a biosynthetic sequence, $\mathrm{CHR} \rightarrow$ cholest-4-en-3-one $\rightarrow$ CHN was demonstrated. When $\mathrm{CHN}$ was utilized as a substrate, 6 -oxoCHN was detected as an enzyme product. Therefore, the conversion of $\mathrm{CHN}$ to 6-oxo$\mathrm{CHN}$ was confirmed. Taken together, the presence of a partial biosynthetic sequence for the early C6-oxidation pathway, $\mathrm{CHR} \rightarrow$ cholest-4-en-3-one $\rightarrow \mathrm{CHN} \rightarrow 6$-oxoCHN, was confirmed in the tomato plants.

The conversion of $\mathrm{CHN}$ to 6-oxoCHN involves the incorporation of an oxygen atom at the C-6 of CHN. ${ }^{7}$ In the late C6-oxidation pathway for $\mathrm{C}_{27}$-BRs biosynthesis in the tomato, the same C6-oxidation from 6-deoxo-28-norCS to 28-norCS has been shown to be catalyzed by a cytochrome P450, designated as $\mathrm{CYP} 85{ }^{7}$, thereby suggesting that the conversion of CHN to 6-oxoCHN can also be catalyzed by CYP85 in the tomato. To evaluate this possibility, enzyme solutions were prepared from a wild-type (Alisa) and a dwarf mutant $(d x)$ with a defective CYP85 gene, and the conversion of $\mathrm{CHN}$ to 6-oxoCHN was assessed with the enzyme solutions using the same enzyme assay as described above. As shown in Figure 2, both enzyme solutions prepared from the wild-type and $d x$ mutant successfully mediated the 6-oxidation of $\mathrm{CHN}$ to 6-oxoCHN, thereby indicating that the 6-oxidation of $\mathrm{CHN}$ to 6-oxoCHN is not catalyzed by CYP85 in the tomato plant.

This study is the first to demonstrate that two sterols--cholest4-en-3-one and 6-oxoCHN--are contained in young tomato plants. Cholest-4-en-3-one and 6-oxoCHN are the counterparts of campest-4-en-3-one and 6-oxoCN in the early C6-oxidation pathway for $\mathrm{C}_{28}$-BRs biosynthesis, respectively (Fig. 1). This suggests that cholest-4-en-3-one and 6-oxoCHN are biosynthesized and metabolized in a manner similar to that of campest4-en-3-one and 6-oxoCN in $\mathrm{C}_{28}$-BRs biosynthesis in plants. Therefore, cholest-4-en-3-one is believed to be generated from
(A)

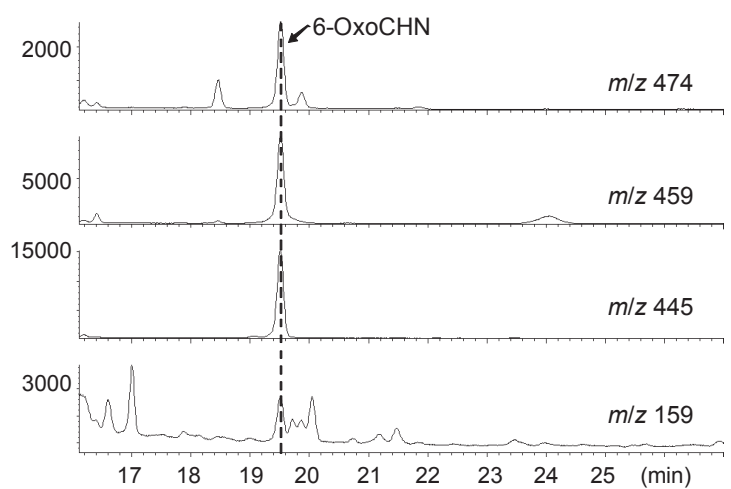

(B)

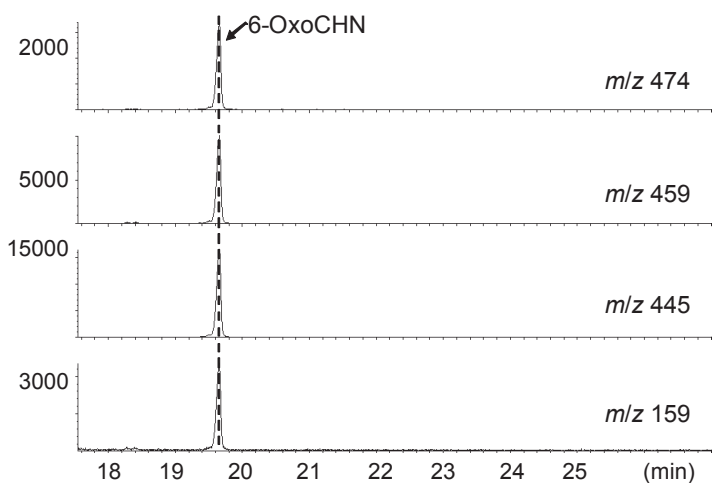

Figure 2. Enzymatic conversion of $\mathrm{CHN}$ to 6-oxoCHN in wild-type (A) and $d x$ mutant (B) of young tomato plants.

CHR via cholest-4-en-3 $\beta$-ol, and converted into CHN via $5 \alpha-$ cholestan-3-one. CHN is believed to be metabolized into 6-oxoCHN via $6 \alpha$-hydroxyCHN in tomato plants.

In vitro enzyme assay using tomato $d x$ mutant shows that CYP85 is not responsible for the 6-oxidation of CHN to 6-oxoCHN in tomato. In Arabidopsis thaliana, two orthologs of tomato CYP85, designated as CYP85A1 and CYP85A2 have been identified. ${ }^{3}$ Molecular genetic and biochemical analysis of CYP85A1 and CYP85A2 revealed that CYP85A1 is a BR 
6-oxidase, and CYP85A2 is a bi-functional enzyme whose activity is not only BR 6-oxidase but also brassinolide (BL) synthase which catalyze the conversion of CS to BL, the last step of C28-BRs biosynthesis. Recently, the same bi-functional enzyme as CYP85A2, designated as CYP85A3, has been characterized in tomato fruit. ${ }^{8}$ We found that both $\mathrm{CYP} 85 \mathrm{~A} 1$ and CYP85A2 are not responsible for the 6-oxidation of $\mathrm{CHN}$ to 6-oxoCHN in Arabidopsis (data published elsewhere), suggesting that CYP85A3 dose not catalyze the 6-oxidation in tomato. However, the final conclusion should be waited until the precise metabolic study will be carried out with tomato cyp $85 a 3$ mutant.

In a previous study, we demonstrated the presence of the late C6-oxidation pathway for $\mathrm{C}_{27}$-BRs in tomato plants. ${ }^{7} \mathrm{In}$ this study, the presence of a partial biosynthetic sequence for the early C6-oxidation pathway for $\mathrm{C}_{27}$-BRs, $\mathrm{CHR} \rightarrow$ cholest4-en-3-one $\rightarrow \mathrm{CHN} \rightarrow 6$-oxoCHN, was confirmed in the plants. Although the downstream biosynthetic sequence from 6-oxo$\mathrm{CHN}$ to 28-norCS has yet to be clearly elucidated, the results of this study indicate that both the early and late C6-oxidation pathway for $\mathrm{C}_{27}$-BRs biosynthesis can generate 28-norCS in tomato plants. In the aid for $S$-adenosyl-methionine and NAD$\mathrm{PH}, 28$-norCS is methylated at $\mathrm{C}$-24 to synthesize active $\mathrm{C}_{28}-\mathrm{BR}$, $\mathrm{CS}$ in tomato plants. ${ }^{7}$ Considering that $\mathrm{CS}$ is more biologically active than 28-norCS, the early and late C6-oxidation pathway for $\mathrm{C}_{27}$-BRs biosynthesis can be concluded to be alternative routes for the synthesis of biologically active $\mathrm{C}_{28}$-BR, CS in the plants.

\section{Experimental Section}

Isolation of 6-oxoCHN and cholest-4-en-3-one in young tomato plants. Young tomato plants $(100 \mathrm{~g})$ were homogenized with $80 \%$ methanol $(1 \mathrm{~L})$, followed by chloroform $(1 \mathrm{~L})$. The chloroform soluble fraction was dried in vacuo, and partitioned between $n$-hexane $(500 \mathrm{~mL})$ and $70 \%$ methanol $(500 \mathrm{~mL})$. The obtained $n$-hexane soluble fraction was concentrated in dryness and unsaponified for 2 hours with $5 \% \mathrm{KOH}$ in $70 \%$ ethanol. After extraction with $n$-hexane, the unsaponified lipids (100 mg) were purified with an $\mathrm{SiO}_{2}(20 \mathrm{~g}$, Merck) column eluted with chloroform containing $0,1,2$, and $3 \%$ methanol (v/v, $200 \mathrm{~mL}$ each). The $1 \%$ methanol in the chloroform fraction was purified further with a Sep-Pak C18 cartridge column eluted with 70 , 80,90 , and $100 \%$ methanol ( $5 \mathrm{~mL}$ each). The $100 \%$ methanol fraction was then subjected to reversed-phase HPLC (SenshuPak Pegasil-B, $4.6 \times 250 \mathrm{~mm}$ ) eluted with aqueous methanol as a mobile phase ( 0 - $10 \mathrm{~min}: 50 \%, 10-20 \mathrm{~min}$ : gradient to $100 \%, 20-70 \mathrm{~min}: 100 \%$ methanol) at a flow rate of $2.5 \mathrm{~mL} /$ min. Fractions were collected every minute. The HPLC fractions corresponding to authentic 6-oxoCHN (18 - $19 \mathrm{~min})$ and cholest-4-en-3-one (37 - $39 \mathrm{~min}$ ) were collected.

Enzyme preparation and assay. Young tomato plants (5 g) were ground in a mortar and pestle with cold $0.1 \mathrm{M}$ sodium phosphate (pH 7.4) buffer containing 15 mM2-mercaptoethanol,
$1 \mathrm{mM}$ EDTA, $1 \mathrm{mM}$ dithiothreitol, $0.1 \mathrm{mM}$ phenylmethylsulfonyl fluoride, $40 \mathrm{mM}$ ascorbate, $250 \mathrm{mM}$ sucrose, and 10\% (v/v) glycerol. The homogenates were centrifuged for $15 \mathrm{~min}$ at $8,000 \mathrm{~g}$ to remove any cell debris. The supernatants were re-centrifuged for $30 \mathrm{~min}$ at $20,000 \mathrm{~g}$. The resultant supernatant was then precipitated via the addition of cold acetone to a final concentration of $40 \%(\mathrm{v} / \mathrm{v})$. The supernatant-acetone mixture was maintained at $-20{ }^{\circ} \mathrm{C}$ for $10 \mathrm{~min}$, then centrifuged for $10 \mathrm{~min}$ at $13,000 \mathrm{~g}$. The resultant precipitate was then dissolved in assay buffer containing $0.1 \mathrm{M}$ sodium phosphate $(\mathrm{pH} 7.4)$ containing $1.5 \mathrm{mM} 2$-mercaptoethanol and 20\% (v/v) glycerol, and used as the cell-free enzyme solution.

The reactions were initiated via the addition of substrates (CHR, cholest-4-en-3-one and CHN), followed by $30 \mathrm{~min}$ of incubation at $37^{\circ} \mathrm{C}$. The metabolites of the enzyme reactions were extracted with ethyl acetate $(1.2 \mathrm{~mL})$ and concentrated in vacuo. The ethyl acetate-soluble fraction was loaded onto a Sep-Pak C18 cartridge column, and washed sequentially with $50 \%$ and $60 \% \mathrm{MeOH}(5 \mathrm{~mL}$ each). The fraction eluted with $100 \% \mathrm{MeOH}$ was concentrated in vacuo, dissolved in $50 \mathrm{uL}$ of $\mathrm{MeOH}$, and then subjected to a reversed-phase HPLC, as mentioned above. Finally, the enzyme products were analyzed via capillary GC-MS/SIM after suitable derivatization.

GC-MS/SIM analysis. GC-MS/SIM analysis was performed a capillary GC-MS: a Hewlett-Packard 5973 mass spectrometer (Electron impact ionization, 70 electron voltage) coupled to 6890 gas chromatography fitted with a fused silica capillary column (HP-5, $0.25 \mathrm{~mm} \times 30 \mathrm{~m}, 0.25 \mu \mathrm{m}$ film thickness). The oven temperature was maintained at $175{ }^{\circ} \mathrm{C}$ for $2 \mathrm{~min}$, elevated to $280{ }^{\circ} \mathrm{C}$ at a rate of $40{ }^{\circ} \mathrm{C} \mathrm{min}^{-1}$ and then maintained at $280{ }^{\circ} \mathrm{C}$. Helium was used as the carrier gas at a flow rate of

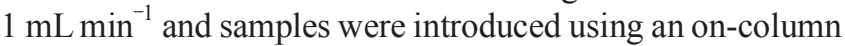
injection mode.

Acknowledgments. This study was supported by the Korea Research Foundation (grant No. KRF-2005-041-C00432) and the Korea Research Foundation (grant No. KRF-2005-015C00471).

\section{Reference}

1. Bishop, G. J.; Yokota, T. Plant Cell Physiol. 2001, 42, 114.

2. Bajguz, A.; Tretyn, A. Phytochemistry 2003, 62, 1027.

3. Kim, T.-W.; Hwang, J.-Y.; Kim, Y.-S.; Joo, S.-H.; Chang, S. C.; Lee, J. S.; Takatsuto, S.; Kim, S.-K. The Plant Cell 2005, 17, 2397.

4. Fujioka, S. In Brassinosteroids: Steroidal Plant Hormones; Sakurai, A., Yokota, T., Clouse, S. D., Eds.; Springer-Verlag: Tokyo, 1999; p 21.

5. Fujioka, S.; Yokota, T. Annu. Rev. Plant Biol. 2003, 54, 137.

6. Kim, S.-K. In Brassinosteroids: Chemistry, Bioactivity and Application; Cultler, H. G., Yokota, T., Adam, G., Eds.; ACS Symposium Series 474: Washington DC, 1991; p 26.

7. Kim, T.-W.; Chang, S. C.; Lee, J. S.; Takatsuto, S.; Yokota, T.; Kim, S.-K. Plant Physiol. 2004, 135, 1231.

8. Nomura, T.; Kushiro, T.; Yokota, T.; Kamiya, Y.; Bishop, G. J.; Yamaguchi, S. J. of Biol. Chem. 2005, 280, 17873. 